\title{
Erythroderma, CTCAE
}

National Cancer Institute

\section{Source}

National Cancer Institute. Erythroderma, CT CAE. NCI Thesaurus. Code C143450.

A disorder characterized by generalized inflammatory erythema and exfoliation. The inflammatory process involves $>90 \%$ of the body surface area. 